\title{
A MOMENT PROBLEM
}

\author{
LAJOS TAKÁCS * \\ (Received 4 February 1965)
}

\section{Introduction}

Let $v$ be a discrete random variable taking on nonnegative integer values and set $\boldsymbol{P}\{\nu=k\}=P_{k}, k=0,1, \cdots$. Suppose that the binomial moments

$$
B_{r}=E\left\{\left(\begin{array}{l}
v \\
r
\end{array}\right)\right\}=\sum_{k=r}^{\infty}\left(\begin{array}{l}
k \\
r
\end{array}\right) P_{k}, \quad r=0,1, \cdots,
$$

are finite. Frequently the problem arises under what conditions the probabilities $P_{k}, k=0,1, \cdots$, can be determined uniquely by the sequence of moments $B_{r}, r=0,1, \cdots$, and how it can be done.

In what follows we shall show that if $\lim _{\sup _{r \rightarrow \infty}} B_{r}^{1 / r}<\infty$, then $\left\{P_{k}\right\}$ can be determined uniquely by $\left\{B_{r}\right\}$ and we shall give an explicit formula for $P_{k}, k=0,1, \cdots$. If $\lim \sup _{r \rightarrow \infty} B_{r}^{1 / r}=\infty$, then, in general, $\left\{P_{k}\right\}$ cannot be determined uniquely by $\left\{B_{r}\right\}$.

\section{An inversion formula}

The probabilities $P_{k}, k=0,1, \cdots$, can be determined in several possible ways, but formula (2) seems to be the most convenient one.

THEOREM. Let $\nu$ be a discrete random variable taking on nonnegative integer values and set $\boldsymbol{P}\{\boldsymbol{v}=k\}=P_{k}, k=0,1, \cdots$. If the binomial moments

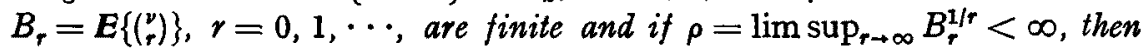

$$
P_{k}=\sum_{r=k}^{\infty} \frac{\left(\begin{array}{l}
r \\
k
\end{array}\right)}{(1+q)^{r+1}} \sum_{j=k}^{r}(-1)^{j-k}\left(\begin{array}{c}
r-k \\
j-k
\end{array}\right) q^{r-j} B_{j}
$$

where $q$ is nonnegative and greater than $\left(\rho^{2}-1\right)$. If, in particular, $\rho=\lim \sup _{r \rightarrow \infty} B_{r}^{1 / r}<1$, then we can always choose $q=0$ and (2) reduces to

$$
P_{k}=\sum_{r=k}^{\infty}(-1)^{r-k}\left(\begin{array}{l}
r \\
k
\end{array}\right) B_{r} .
$$

- This research was supported by the Office of Naval Research under Contract Number Nonr-266(59), Project Number 042-205. 
Proof. The generating function

$$
P(z)=\sum_{j=0}^{\infty} P_{1} z^{j}
$$

is uniformly convergent in the circle $|z|<1$ and $P(z)$ is regular if $|z|<1$. Hence

$$
P^{(k)}(z)=\frac{d^{k} P(z)}{d z^{k}}=k ! \sum_{j=k}^{\infty}\left(\begin{array}{l}
j \\
k
\end{array}\right) P_{j} z^{j-k}
$$

for $k=0,1, \cdots$ and $|z|<1$. If $z=0$ in (5), then we get

$$
P_{k}=\frac{1}{k !} P^{(k)}(0) \text {. }
$$

Thus the problem of finding $P_{k}$ can be reduced to finding $P(z)$ in a neighborhood of $z=0$.

If $B_{r}$ is finite, then

$$
B_{r}=\frac{1}{r !}\left(\frac{d^{r} P(z)}{d z^{r}}\right)_{z=1}
$$

and for $|z-1|<1 / \rho$

$$
P(z)=\sum_{r=0}^{\infty} B_{r}(z-1)^{r}
$$

The right hand side of $(8)$ is uniformly convergent in the circle $|z-1|<1 / \rho$ and $P(z)$ is regular if $|z-1|<1 / \rho$. Hence

$$
P^{(k)}(z)=\frac{d^{k} P(z)}{d z^{k}}=k ! \sum_{r=k}^{\infty}\left(\begin{array}{l}
r \\
k
\end{array}\right) B_{r}(z-1)^{r-k}
$$

for $k=0,1, \cdots$, and $|z-1|<1 / \rho$.

If $\rho<1$, and we put $z=0$ in (9), then by (6) we get (3). We note that (3) is an oscillating series which is convergent if and only if $\lim _{r \rightarrow \infty} r^{k} B_{r}=0$.

If $\rho<\infty$, then (9) is a regular function of $z$ in the circle $|z-1|<1 / \rho$. By analytical continuation we can extend the definition of (9) to the domain $|z|<1$ and in this domain (9) agrees with (5). Now we shall show that the definition of (9) can easily be extended to a neighborhood of $z=0$ by using Euler's transformation of series. (Cf. Hardy [2], Chapter VIII.) Let $q \geqq 0$ and form the $E_{q}$-transform of (9),

$$
P_{q}^{(k)}(z)=k ! \sum_{r=k}^{\infty} \frac{1}{(1+q)^{r+1}} \sum_{j=0}^{r}\left(\begin{array}{l}
r \\
j
\end{array}\right)\left(\begin{array}{l}
j \\
k
\end{array}\right) q^{r-j}(z-1)^{j-k} B_{j} .
$$

For $|z-1|<1 / \rho$ we have $P_{a}^{(k)}(z)=P^{(k)}(z)$ given by (9) because Euler's transformation is consistent. Now by using a theorem of Knopp [6] we can 
establish the domain $R_{q}$ in which (10) is convergent and represents a regular function of $z$. Suppose that $P_{q}^{(k)}(z)$ is analytically extended along every ray of origin $z=1$ until we reach the first singular point (if any) of $P_{q}^{(k)}(z)$ on the ray. Denote by $\Gamma$ the set of all singular points obtained in this way. Then $R_{a}$ can be represented as the set of points common to all the circles $|z-1+q(\gamma-1)|<(1+q)|\gamma-1|$ for $\gamma \in \Gamma$. Evidently $|\gamma-1| \geqq 1 / \rho$ and $|\gamma| \geqq 1$ for all $\gamma \in \Gamma$ and there exists a $\gamma \in \Gamma$ such that $|\gamma-1|=1 / \rho$. Hence it follows that $R_{q}$ always contains the point $z=0$ if $q>\left(\rho^{2}-1\right)$ and $R_{q}$ never contains $z=0$ if $q \leqq(\rho-1) / 2$. For example, if $|\gamma| \geqq(1+\rho) / \rho$ for every $\gamma \in \Gamma$, then we can choose $q$ as any nonnegative number greater than $(\rho-1) / 2$, however, if $\Gamma$ contains a $\gamma$ for which $|\gamma-1|=1 / \rho$ and $|\gamma|=1$, then $q$ must be chosen greater than $\left(\rho^{2}-1\right)$ in order that $R_{q}$ contain $z=0$. Accordingly if $q>\left(\rho^{2}-1\right)$, then in some neighborhood of $z=0$ we have $P_{q}^{(k)}(z)=P^{(k)}(z)$ given by $(5)$. Thus by (6) we have $P_{k}=P_{q}^{(k)}(0) / k !, k=0,1, \cdots$, which yields (2).

\section{Examples}

(i) Suppose that $B_{r}=E\left\{\left(\begin{array}{l}r \\ r\end{array}\right)\right\}=a^{r} / r !, r=0,1, \cdots$, where $a$ is a positive number. Then $\lim _{r \rightarrow \infty} B_{r}^{1 / r}=0$ and $\rho=0$. By (3)

$$
P_{k}=\sum_{r=k}^{\infty}(-1)^{r-k}\left(\begin{array}{l}
r \\
k
\end{array}\right) \frac{a^{r}}{r !}=e^{-a} \frac{a^{k}}{k !}, \quad k=0,1, \cdots .
$$

(ii) Suppose that $B_{r}=E\left\{\left(\begin{array}{l}\nu \\ r\end{array}\right)\right\}=a^{r}, r=0,1, \cdots$, where $a$ is a positive number. Then $\lim _{r \rightarrow \infty} B_{r}^{1 / r}=a$ and $\rho=a$. If $a<1$, then we can apply formula (3) to obtain

$$
P_{k}=\sum_{r=k}^{\infty}(-1)^{r-k}\left(\begin{array}{l}
r \\
k
\end{array}\right) a^{r}=\frac{a^{k}}{(1+a)^{k+1}}, \quad k=0,1, \cdots
$$

If $a<\infty$, then we obtain by (2) that

$$
P_{k}=\sum_{r=k}^{\infty}\left(\begin{array}{l}
r \\
k
\end{array}\right) \frac{a^{k}(q-a)^{r-k}}{(1+q)^{r+1}}=\frac{a^{k}}{(1+a)^{k+1}}, \quad k=0,1, \cdots,
$$

where $q>(a-1) / 2$. The best choice is $q=a$.

(iii) Let $A_{1}, A_{2}, \cdots, A_{n}, \cdots$ be an infinite sequence of events. Denote by $v$ the number of events occurring among $A_{1}, A_{2}, \cdots, A_{n}, \cdots$ It can easily be seen that

$$
B_{r}=E\left\{\left(\begin{array}{l}
\nu \\
r
\end{array}\right)\right\}=\sum_{1 \leq i_{1}<i_{2}<\cdots<i_{r}<\infty} \boldsymbol{P}\left\{A_{i_{1}} A_{i_{2}} \cdots A_{i_{r}}\right\} .
$$

If $\rho=\lim \sup _{r \rightarrow \infty} B_{r}^{1 / r}<1$, then the probability that exactly $k$ events 
occur among $A_{1}, A_{2}, \cdots, A_{n}, \cdots$ is given by (3). Formula (3) was found first by Jordan [3], [4], [5], for the case when $A_{n+1}=A_{n+2}=\cdots=0$, the impossible event. (Cf. also [8].)

If $\rho=\lim \sup _{r \rightarrow \infty} B_{r}^{1 / r}<\infty$, then the probability that exactly $k$ events occur among $A_{1}, A_{2}, \cdots, A_{n}, \cdots$ is given by (2) with $q>\left(p^{2}-1\right)$. In some particular cases we can choose $q>(p-1) / 2$.

(iv) Consider the previous example. The probability that at least one event occurs among $A_{1}, A_{2}, \cdots, A_{n}, \cdots$ is given by $\boldsymbol{P}\left\{A_{1}+A_{2}+\cdots+A_{n}+\right.$ $\cdots\}=1-P_{0}$. If $\rho<1$, then by (3)

$$
\boldsymbol{P}\left\{A_{1}+A_{2}+\cdots+A_{n}+\cdots\right\}=\sum_{r=1}^{\infty}(-1)^{r-1} B_{r} .
$$

If $\rho<\infty$, then by (2)

$$
\boldsymbol{P}\left\{A_{1}+A_{2}+\cdots+A_{n}+\cdots\right\}=1-\sum_{r=0}^{\infty} \frac{1}{(1+q)^{r+1}} \sum_{j=0}^{r}(-1)^{j}\left(\begin{array}{l}
r \\
j
\end{array}\right) q^{r-j} B_{j}
$$

where $q>\left(\rho^{2}-1\right)$. In some particular cases we can choose $q>(\rho-1) / 2$.

Formula (15) was found first by Poincare [7] for the case when $A_{n+1}=A_{n+2}=\cdots=0$, the impossible event. Dvoretzky [1] proved that (15) holds if $\lim _{r \rightarrow \infty} B_{r}=0$.

(v) It is interesting to mention also the following simple example. A balanced coin is tossed repeatedly. We say that event $A_{n}$ occurs if head does not appear among the first $n$ tossings. Denote by $v$ the number of events occurring among $A_{1}, A_{2}, \cdots, A_{n}, \cdots$ By (14) $B_{r}=\boldsymbol{E}\left\{\left(\begin{array}{c}\boldsymbol{r} \\ r\end{array}\right)\right\}=1$ for $r=0,1, \cdots$. In this case (3) is divergent, but by (2) with $q>0$ we get that $P_{k}=\boldsymbol{P}\{\nu=k\}=1 / 2^{k+1}$ for $k=0,1, \cdots$, in agreement with a direct calculation.

\section{References}

[1] Dvoretzky, A. (1963) On an elementary identity in the theory of probability. Columbia University Report, November 13, 1963.

[2] Hardy, G. H. (1949) Divergent Series. Oxford University Press.

[3] Jordan, K. (1927) A valószinűségszámitás alapfogalmai. (Les fondements du calcul des probabilités.) Mathematikai és Physikai Lapok 34, 109-136.

[4] Jordan, Ch. (1934) Le théorème de probabilité de Poincaré, généralisé au cas de plusieurs variables indépendantes. Acta Sci. Math. (Szeged) 7, 103-111.

[5] Jordan, Ch. (1939) Problèmes de la probabilité des épreuves répétées dans le cas général. Bull. de la Société Mathématique de France 67, 223-242.

[6] Knopp, K. (1923) Uber das Eulersche Summierungsverfahren I-II. Mathem. Zeitschr. 15 (1922), 226-253 and $18(1923), 125-156$.

[7] Poincaré, H. (1896) Calcul des Probabilités. Gauthier-Villars, Paris.

[8] Takács, L. (1958) On a general probability theorem and its applications in the theory of stochastic processes. Proc. Cambridge Phil. Soc. 54, 219-224.

Department of Mathematical Statistics

Columbia University 